\title{
Angular displacement and velocity sensors based on electric-LC (ELC) loaded microstrip lines
}

\author{
Jordi Naqui, Student Member IEEE, and Ferran Martín, Fellow IEEE
}

\begin{abstract}
Planar microwave angular displacement and angular velocity sensors implemented in microstrip technology are proposed. The transducer element is a circularly-shaped divider/combiner, whereas the sensing element is an electric-LC (ELC) resonator, attached to the rotating object and magnetically coupled to the circular (active) region of the transducer. The angular variables are measured by inspection of the transmission characteristics, which are modulated by the magnetic coupling between the resonator and the divider/combiner. The degree of coupling is hence sensitive to the angular position of the resonator. As compared to coplanar waveguide (CPW) angular displacement and velocity sensors, the proposed microstrip sensors do not require air bridges, and the ground plane provides backside isolation.
\end{abstract}

Index Terms - Angular sensors, microstrip technology, microwave sensors, rotation speed sensors.

\section{INTRODUCTION}

$\mathrm{M}^{\mathrm{s}}$ ICROWAVE sensors based on split rings that deal with the measurement of spatial variables (e.g., alignment or displacement) are increasingly popular [1]-[7]. These sensors usually consist of a transmission line coupled to a resonator, and the relative motion between these two elements is measured through the transmission characteristics of an $\mathrm{RF} /$ microwave signal. The sensing principle in [2]-[7] relies on symmetry properties; typically, in transmission lines symmetrically loaded with resonators, line-to-resonator coupling is prevented, and the lines exhibit total transmission. However, by breaking the symmetry through the effects of a spatial variable (e.g., a rotation), a transmission zero emerges, and the spatial variable can thus be inferred.

This paper proposes angular displacement and velocity sensors consisting of a microstrip line loaded with an electric-LC (ELC) resonator [8], based on the aforementioned symmetry properties. The proposed implementation is an alternative to the coplanar waveguide (CPW) microwave sensors reported in

Manuscript received October 18, 2013. This work has been supported by projects TEC2010-17512, EMET CSD2008-00066, TEC2011-13615-E, 2009SGR-421 and by the FPU Grant AP2010-0431.

J. Naqui and F. Martín are with GEMMA/CIMITEC, Departament d'Enginyeria Electrònica, Universitat Autònoma de Barcelona, 08193 Bellaterra, Spain. E-mail: Ferran.Martin@uab.es.
[7], and particularly suited in systems that require backside isolation. A discussion on the performance of the considered symmetry-based approach can be found in [7].

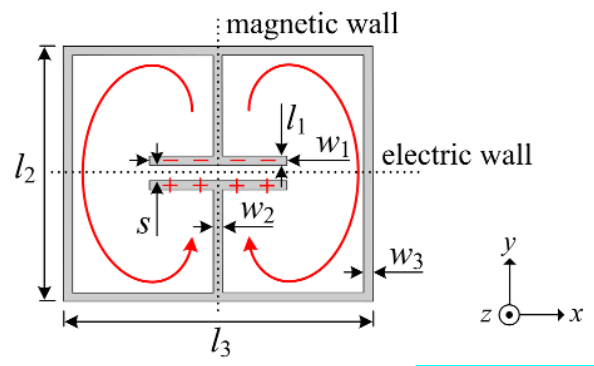

Fig. 1. Typical topology of an ELC resonator. A sketch of the current and charge pattern at the fundamental resonance is indicated.

\section{PRINCIPLE OF OPERATION}

An ELC resonator is composed of two connected metallic loops with a common gap (Fig. 1). The resonator is bisymmetric, and exhibits electric and magnetic walls in orthogonal planes at the fundamental resonance. Fig. 2 illustrates an ELC resonator symmetrically etched between a pair of parallel microstrip lines. Let us consider that the line pair is fed by a common-mode signal, so that the symmetry plane (i.e., the midplane) behaves as a magnetic wall. As proven in [9], the line is transparent to signal transmission if the ELC electric wall is aligned with the symmetry plane of the lines [Fig. 2(a)]. Contrarily, a transmission zero arises if the alignment is with the ELC magnetic wall [Fig. 2(b)]. In the former situation, the fundamental ELC resonance cannot be excited. However, the resonator is strongly coupled (magnetically) to the lines if the magnetic walls are aligned. Such behavior, explained through equivalent circuit models in [9], suggests that rotations with a $90^{\circ}$ dynamic range can be measured from the common-mode transmission coefficient.

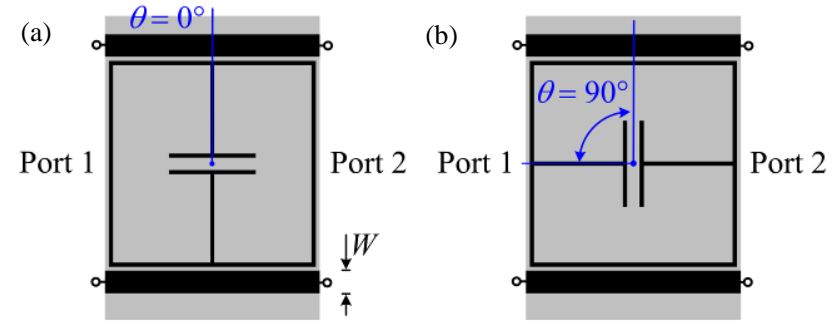

Fig. 2 Parallel microstrip lines loaded with an ELC resonator for the two extreme cases of angular orientation; (a) $\theta=0^{\circ}$ and (b) $\theta=90^{\circ}$. 
(a)

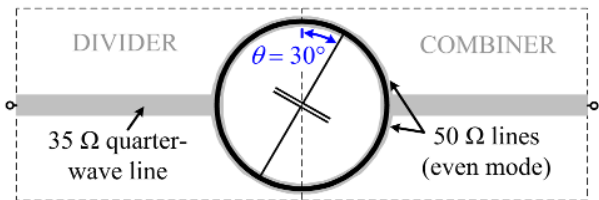

(b)

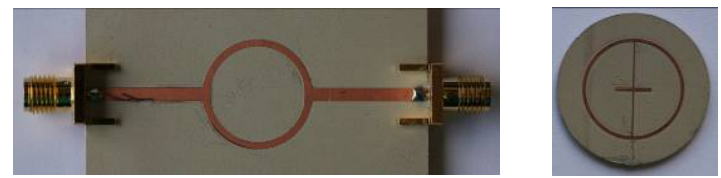

Fig. 3. Angular displacement sensor. (a) Layout, and (b) photograph. The substrates are Rogers RO3010 with dielectric constant $\varepsilon_{r}=11.2$, thickness $h=1.27 \mathrm{~mm}$ (microstrip line) and $h=0.635 \mathrm{~mm}$ (resonator), and loss tangent $\tan \delta=0.0023$. The width $W$ of the $35.35 \Omega$ inverters is $2.06 \mathrm{~mm}$, and that of the $50 \Omega$ lines is $1.04 \mathrm{~mm}$; ELC mean radius $r_{0}=8.05 \mathrm{~mm}, w_{1}=6 \mathrm{~mm}$, $w_{2}=l_{1}=s=0.2 \mathrm{~mm}$, and $w_{3}=0.5 \mathrm{~mm}$.

\section{ANGULAR DISPLACEMENT AND VELOCITY SENSORS}

An angular displacement sensor inspired on the ELC-loaded parallel microstrip line pair operating in common-mode was designed (Fig. 3). However, rather than using a common-mode source, a T-junction power divider cascaded to another one acting as a combiner was used. Thus, by feeding the structure with a single-ended signal, the divider acts as a single-ended to common-mode signal transition. Conversely, the combiner converts the common-mode signal into a single-ended signal. The circularly-shaped lines exhibit $50 \Omega$ even-mode characteristic impedance. The combiner/divider is implemented with $35.35 \Omega$ impedance inverters (quarter wavelength lines at $1.45 \mathrm{GHz}$ ) to achieve matching to $50 \Omega$ reference ports. The circular $50 \Omega$ line pair (which is the active part of the transducer and hence responsible for resonator coupling) must be arranged face-to-face with the ELC external ring in order to enhance electromagnetic coupling. Furthermore, the line pair as well as the resonator were circularly-shaped to enhance linearity.

Undesired common-to-differential mode conversion may arise for asymmetric loadings (i.e., $0^{\circ}<\theta<90^{\circ}$ ). Mode conversion in [7] was cancelled by air bridges cascaded at both sides of the ELC. In the present implementation, the divider/combiner indeed suppresses the differential mode. However, if the circularly-shaped lines are electrically long, mode mixing is generated along them. In order to reduce electrically the size of these lines at resonance, the electrical size of the resonator was decreased by using a high dielectric constant substrate $\left(\varepsilon_{r}=11.2\right)$. The displacement measurement was performed following the procedure in [7] [see Fig. 4(a)]. The ELC substrate was attached to a step motor through a Teflon slab with $3.5 \mathrm{~mm}$ thickness and dielectric constant $\varepsilon_{r}=2.08$. The air gap between the ELC and the microstrip lines was set to $0.254 \mathrm{~mm}$. As shown in Fig. 4(b), the rotation angle can be sensed from the notch magnitude and frequency, being the dependence reasonably linear.

Let us now consider that the ELC is continuously rotating. To obtain the angular velocity, $\omega_{r}$, the set-up reported in [7] was used. Specifically, a harmonic signal tuned at the notch frequency corresponding to $\theta=10^{\circ}\left(f_{0}=1.308 \mathrm{GHz}\right)$ was injected to the input port, whereas an isolator plus an envelope detector were cascaded to the output port. The step motor was configured with $f_{r}=1 \mathrm{~Hz}$ and $50 \mathrm{~Hz}\left(\omega_{r}=2 \pi f_{r}\right)$. The envelope signals (which are dependent on the transmission coefficient) were captured by an oscilloscope (Fig. 5), and the velocities were derived from the time difference between consecutive transmission peaks $T_{m}=T_{r} / 2=1 / 2 f_{r}$. The measured velocities $\left(f_{r}=0.998 \mathrm{~Hz}\right.$ and $50.251 \mathrm{~Hz}$ ) validate the approach.

In summary, a novel microstrip sensor to measure angular displacements and velocities has been proposed. Rotation speeds as high as required can be measured, and precision can be improved by averaging the time between peaks.
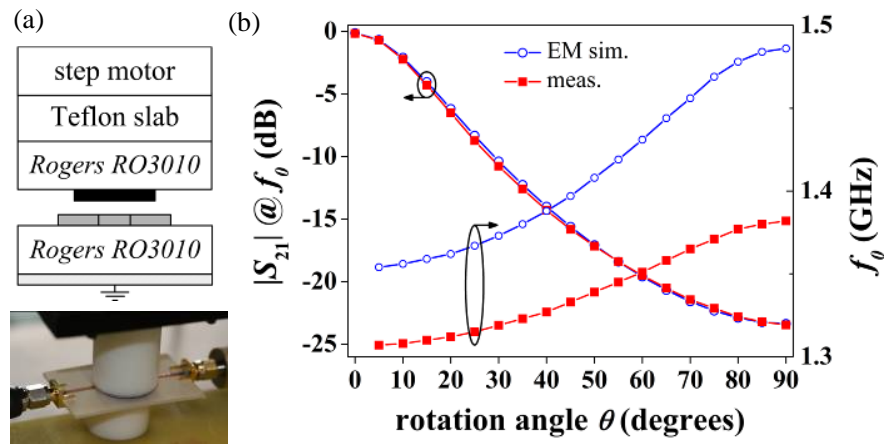

Fig. 4. (a) Experimental set-up, and (b) notch magnitude and frequency versus the angular displacement. The frequency shift is attributed to substrate and fabrication tolerances ( $f_{0}$ is very sensitive to the resonator parameters).

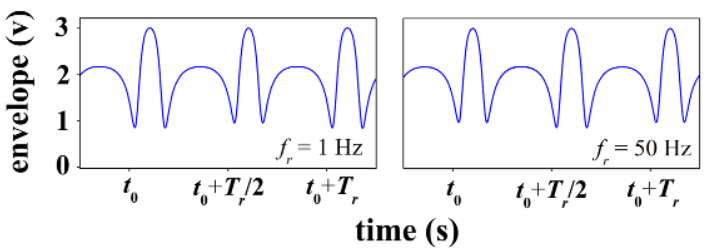

Fig. 5 Angular velocity measurements for an arbitrary initial time $t_{0}$.

\section{REFERENCES}

[1] C. Mandel, B. Kubina, M. Schüßler, R. Jakoby, "Passive chipless wireless sensor for two dimensional displacement measurement”, 41st Europ. Microw. Conf., Manchester (UK), Oct. 2011, pp. 79-82.

[2] J. Naqui, M. Durán-Sindreu, F. Martín, “Alignment and position sensors based on split ring resonators”, Sensors, vol. 12, pp. 11790-11797, Aug. 2012.

[3] J. Naqui, M. Durán-Sindreu, F. Martín, “On the symmetry properties of coplanar waveguides loaded with symmetric resonators: analysis and potential applications”, IEEE MTT-S Int. Microwave Symp., Montreal (Canada), Jun. 2012.

[4] A. Horestani, C. Fumeaux, S. Al-Sarawi, D. Abbott, "Displacement sensor based on diamond-shaped tapered split ring resonator," IEEE Sensors Journal, vol. 13, no. 4, pp. 1153 - 1160, Apr. 2013.

[5] A. Horestani, D. Abbott, C. Fumeaux, "Rotation sensor based on hornshaped split ring resonator,” IEEE Sensors Journal, no. 99, May 2013.

[6] J. Naqui, M. Durán-Sindreu, F. Martín, "Transmission Lines Loaded with Bisymmetric Resonators and Applications," IEEE MTT-S Int. Microwave Symp., Seattle (USA), Jun. 2013.

[7] J. Naqui, F. Martín, "Transmission lines loaded with bisymmetric resonators and their application to angular displacement and velocity sensors,“ IEEE Trans. Microw. Theory Techn., vol. 61, no. 12, pp. 4700-4713, Dec. 2013.

[8] D. Schurig, J. J. Mock, D. R. Smith "Electric-field-coupled resonators for negative permittivity metamaterials." Appl. Phys. Lett., vol. 88, paper 041109, 2006.

[9] J. Naqui, M. Durán-Sindreu, F. Martín, "Selective mode suppression in microstrip differential lines by means of electric-LC (ELC) and magnetic-LC (MLC) resonators,” Appl. Phys. A, to be published. 